Int. J. Odontostomat., 11(1):13-18, 2017.

\title{
Detección de Porphyromonas gingivalis en Pacientes Adultos con Periodontitis Crónica
}

\author{
Porphyromonas gingivalis Detection in Adult Patient with Chronic Periodontitis
}

\author{
Arce Paniagua Marion; Ulloa Carmona Marcelo; Pozo Hernández Patricia² \& Bravo Bown Joel ${ }^{1}$
}

\begin{abstract}
ARCE, M; ULLOA, M; POZO, P \& BRAVO-BOWN, J. Detección de Porphyromonas gingivalis en pacientes adultos con periodontitis crónica. Int. J. Odontostomat., 11(1):13-18, 2017.

RESUMEN: Se realizó la detección de Porphyromonas gingivalis ( $P$. gingivalis) en pacientes adultos con periodontitis crónica que se atendieron en la Clínica de Periodoncia de la Universidad de Antofagasta, a través de técnicas de microbiología convencional y técnica de biología molecular. fueron obtenidas muestras de fluido gingival crevicular de 37 pacientes con periodontitis crónica, con conos de papel $\left(N^{\circ} 30\right)$ por 20 segundos del sitio periodontal más severo de cada cuadrante, posteriormente se realizó cultivo microbiológico en agar sangre y se detectó la presencia de $P$. gingivalis a través de técnicas de microbiología convencional (lupa estereoscópica y fluorescencia) y técnica de biología molecular (reacción en cadena de la polimerasa). se detectó con lupa estereoscópica colonias pigmentadas de negro en el 75,7\% de las muestras $(n=28)$, de ellas el 67,9 \% fueron positivas para $P$. gingivalis con la técnica de fluorescencia $(n=19)$. A través de la reacción en cadena de la polimerasa se pudo confirmar que el $84,2 \%$ de las colonias positivas por fluorescencia correspondían a $P$. gingivalis $(\mathrm{n}=16)$. En la población de estudio analizada es posible concluir que existe una alta prevalencia de $P$. gingivalis en la microbiota subgingival de los pacientes con periodontitis crónica lo que ha sido detectado a través de técnicas de microbiología convencional y mediante la reacción en cadena de la Polimerasa. Los pacientes fumadores presentaron mayor presencia de $P$. gingivalis que los no fumadores.
\end{abstract}

PALABRAS CLAVE: Periodontitis crónica, Porphyromonas gingivalis, Reacción en cadena de la polimerasa.

INTRODUCCIÓN

Porphyromonas gingivalis ( $P$. gingivalis) es una bacteria Gram-negativa anaeróbica estricta pigmentada de negro (Darveau et al., 1997), miembro fundamental de la microbiota patógena en varias enfermedades periodontales caracterizadas por la pérdida de hueso alveolar (Holt \& Ebersole, 2005). Se ha demostrado que este microorganismo posee la capacidad de adherirse a una diversidad de tejidos, entre ellos los tejidos periodontales, invadir las células anfitrionas y multiplicarse. Para lograr esto utiliza diferentes componentes bacterianos como son: fimbrias, proteasas, hemaglutininas y lipopolisacáridos (LPS) (Holt et al., 1999). Es importante observar la prevalencia de este patógeno periodontal por su alta patogenicidad y capacidad infectiva. Las discrepancias encontradas en los estudios de prevalencia de distintas localidades geográficas se han explicado por diferencias étnicas y de hábitos o costumbres (Zhao et al., 2007).
En Antofagasta no se han realizado estudios de detección de $P$. gingivalis en los pacientes con enfermedad periodontal, y dadas las discrepancias antes mencionadas es importante comenzar a desarrollar estudios sobre esta materia.

El objetivo general del estudio fue detectar bacteria $P$. gingivalis en un grupo de pacientes adultos con periodontitis crónica atendidos en el Departamento de Odontología de la Universidad de Antofagasta, Chile. Además, se plantearon los siguientes obsejtivos específicos: detectar mediante técnicas de microbiología convencional (fluorescencia y morfología colonial) la presencia de $P$. gingivalis; detectar mediante técnica de biología molecular (reacción en cadena de la polimerasa) la presencia de $P$. gingivalis y determinar diferencias en la detección de $P$. gingivalis según hábito tabáquico. 


\section{MATERIAL Y MÉTODO}

Diseño del estudio. Se realizó un estudio clínico de naturaleza observacional prospectivo transversal y descriptivo, en el cual se recolectó muestras de fluido gingival crevicular de 20 pacientes adultos diagnosticados con periodontitis crónica y que cumplían con los criterios de inclusión.

\section{Criterios de inclusión.}

- Pacientes adultos de 18 a 65 años de edad atendidos en Clínica de Periodoncia de la Universidad de Antofagasta.

- Presencia de periodontitis crónica (sacos periodontales $\geq a 5 \mathrm{~mm}$ y pérdida en el nivel de inserción clínico $\geq$ a $3 \mathrm{~mm}$ )

- Sin tratamiento antibiótico ni terapia periodontal en los últimos 6 meses.

Consideraciones éticas. Todos los pacientes firmaron un consentimiento informado (previamente validado por el Comité de Ética de la Universidad de Antofagasta).

Toma de muestras y recolección de los datos. Se utilizó una ficha clínica especial para la recolección de los datos. Después de aislar el área con torundas de algodón y secar cuidadosamente con aire, se eliminó la placa supragingival con cureta estéril y se tomó una muestra de fluido gingival crevicular, utilizando dos conos de papel estéril ( $\left.\mathrm{N}^{\circ} 35\right)$ por 20 segundos (Fig. 1). El sitio elegido fue aquel que presentó una mayor

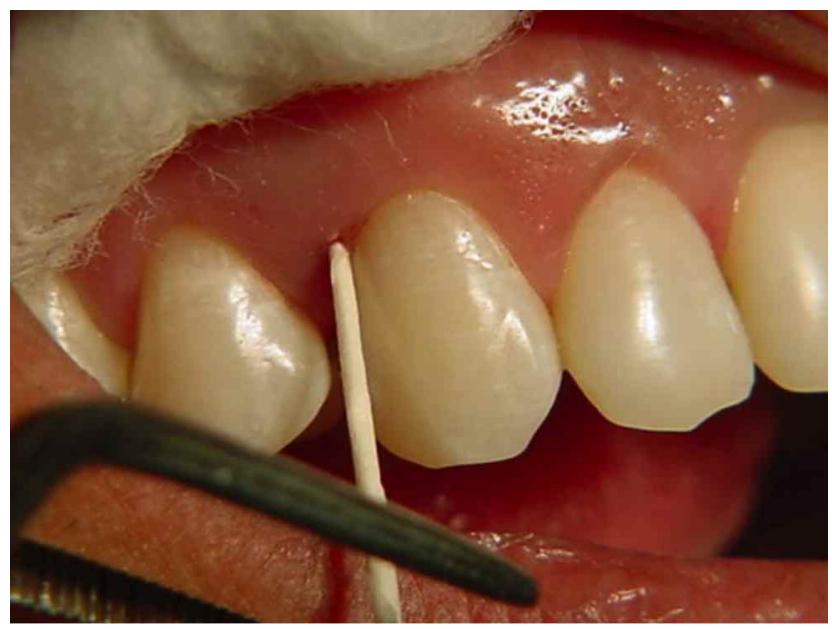

Fig. 1.Técnica de obtención de muestra microbiológica con cono de papel. Se aisla la zona con torundas de algodón, se eliminar la placa bacteriana con cureta, se seca con jeringa triple, se introduce un cono de papel estéril $\left(n^{\circ} 30\right)$ en el surco gingival por 20 segundos. profundidad de sondaje en cada cuadrante. Los conos se depositaron en un tubo eppendorf con 1 $\mathrm{ml}$. de medio de transporte RTF (fluido de transporte reducido) frío sin EDTA (Fig. 2), los que fueron transportados inmediatamente al Laboratorio del Departamento de Odontología, a una temperatura de $4{ }^{\circ} \mathrm{C}$ (en hielo), para su posterior siembra antes de 2 horas.

\section{Cultivo Microbioló-} gico. Las muestras de placa subgingival fueron dispersadas y mezcladas por $45 \mathrm{se}-$ gundos agitando en un vórtex-mixer, seguido de diluciones seriadas en PBS (buffer fosfato $\mathrm{pH} 7,4$ ) de la suspensión bacteriana mantenida en el RTF. Un volumen de $100 \mu$ de la dilución adecuada $\left(10^{-3}\right)$, se sembró en agar sangre. Las placas fueron incubadas en condiciones de anaerobiosis a $35^{\circ} \mathrm{C}$ hasta 14 días, en jarra con generador de atmósfera anaerobia (Anaerogen) (Fig. 3).

Técnicas de Microbiología convencional. Una vez transcurrido el período de incubación se efectuó un análisis para la diferenciación entre los géneros Prevotella

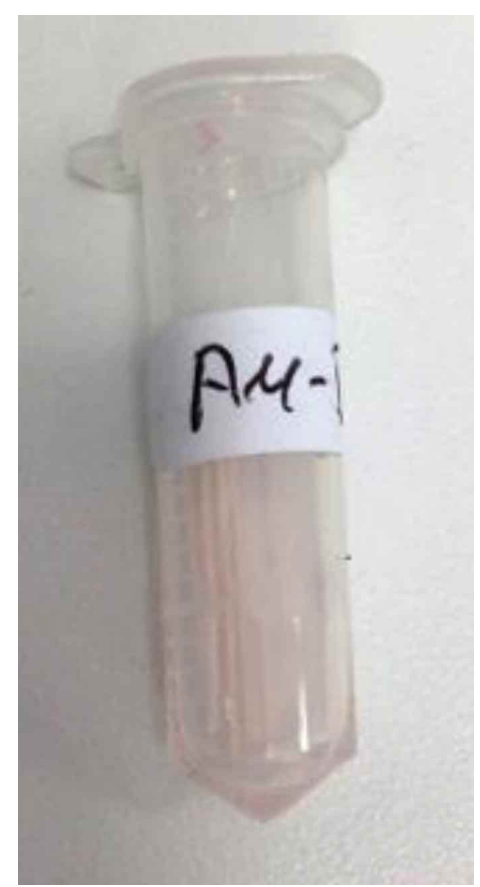

Fig. 2. Medio de transporte en eppendorf. La muestra microbiológica es transportada al laboratorio en un tubo eppendorf que contiene RTF.

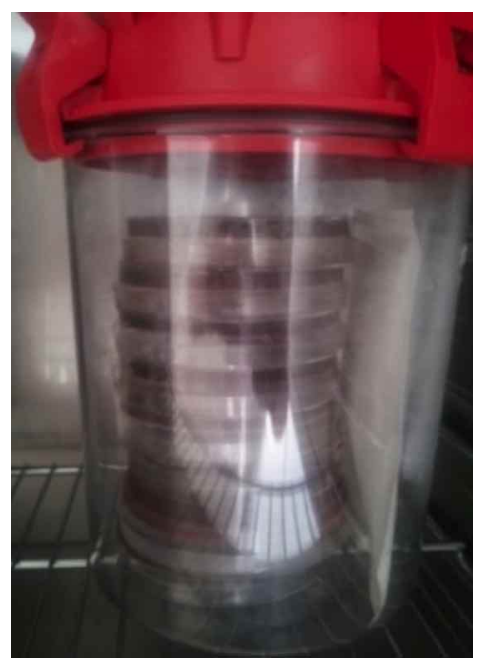

Fig. 3. Siembra Microbiológica. Posterior a la realización de las diluciones seriadas, se siembra en medios de cultivo agar sangre hemina menadiona, las placas de cultivo son mantenidas en una jarra de anaerobiosis con generador de atmósfera anaerobia. 
Porphyromonas. Las placas se sometieron a la acción directa de Luz UV de onda larga $(360 \mathrm{~nm})$ suspendidos en metanol puro. La presencia de fluorescencia negativa (color negro), indica que los microorganismos pertenecen al género Porphyromonas (Fig. 4). Posteriormente se efectuó un análisis macroscópico bajo lupa estereoscópica, para identificar las colonias pigmentadas de negro (Fig. 5). Se escogieron 8 colonias bacterianas pigmentadas de negro por paciente, en lo posible eligiendo cada colonia con diferente morfología. Inmediatamente fueron resembradas cada una por separado en placas de agar sangre heminamenadiona no selectivo e incubadas en condiciones de anaerobiosis a $35^{\circ} \mathrm{C}$ durante 7 a 10 días (Fig. 6).

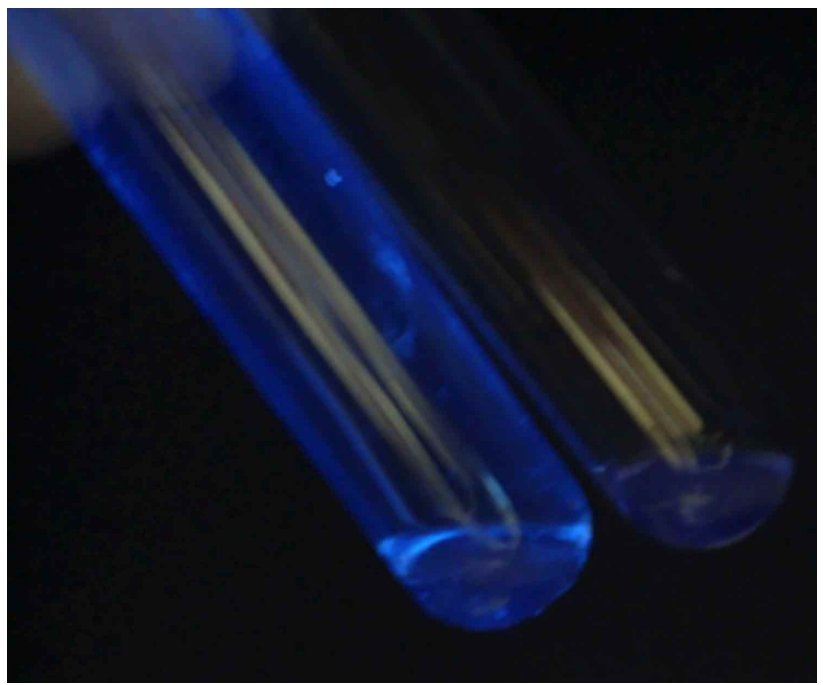

Fig. 4. Técnica de Fluorescencia. Una vez obtenidas las colonias pigmentadas de negro se deposita una o dos en un tubo de ensayo con metanol y se hace incidir luz UV $360 \mathrm{~nm}$. Las colonias que presenten fluorescencia negativa son $P$. gingivalis y las con fluorescencia positiva Prevotella intermedia.

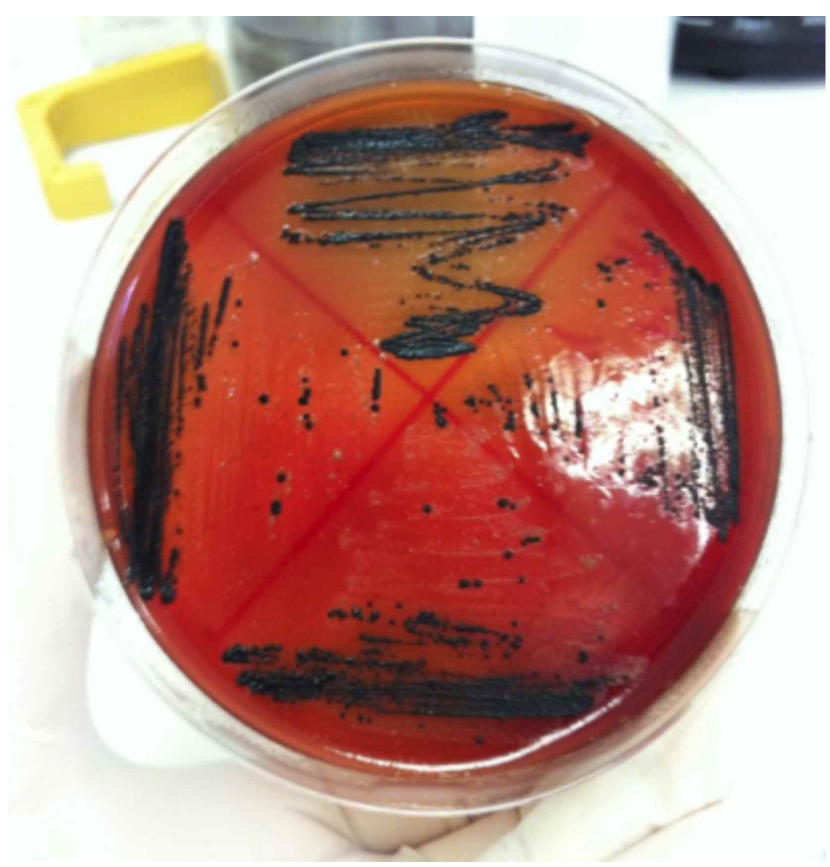

Fig. 6. Recultivo de colonias de $P$. gingivalis de diferente morfología. Se tomaron 8 colonias pigmentadas de negro de distinta morfología y fueron resembradas cada una por separado en placas de agar sangre hemina-menadiiona no selectivo.

La cuantificación de bacterias se realizó mediante la observación del número de colonias encontradas en las placas de agar bajo lupa estereoscópica y a partir de ésta medida se determinó la microbiota total cultivable, expresándolo como unidades formadoras de colonia (UFC) por ml de medio de transporte (RTF).
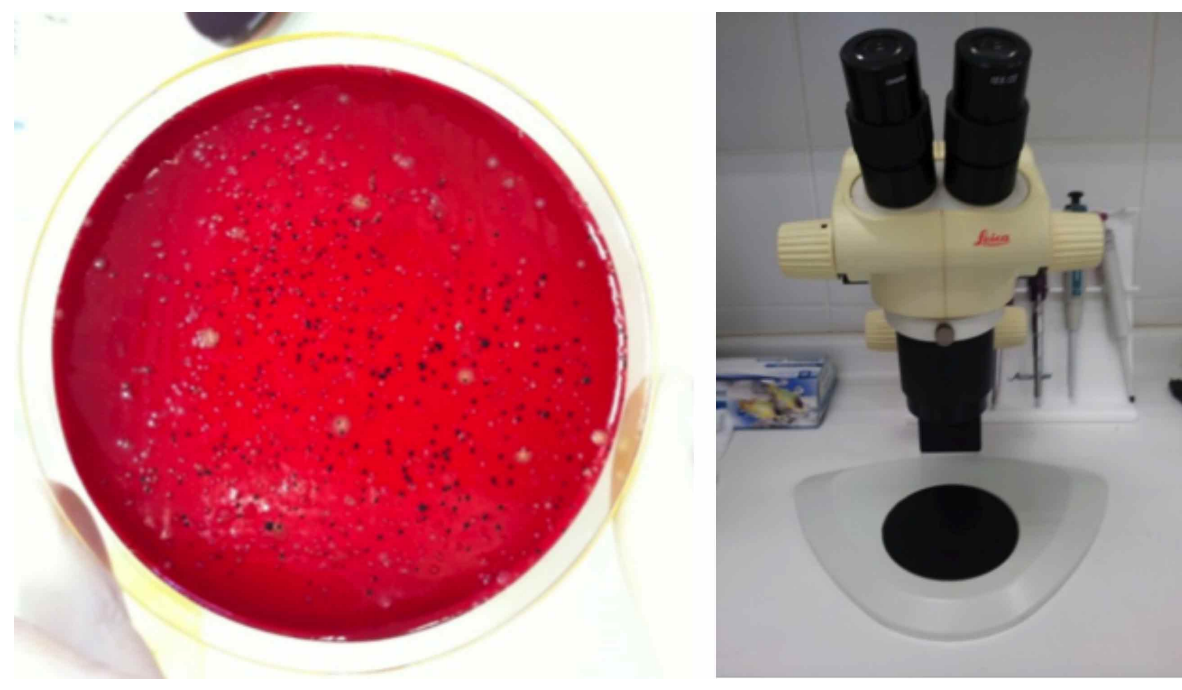

Fig. 5. Placa de cultivo agar sangre menadiona con colonias de microorganismos. Las placas de cultivo fueron analizadas con lupa estereoscópica para detección de bacterias pigmentadas de negro. 


\section{Técnica de Biología Molecular}

Extracción de DNA. Se realizó la extracción de DNA bacteriano desde los recultivos de $P$. gingivalis de acuerdo al protocolo propio de un kit comercial (Genomic DNA Purification Kit) especializado en bacterias y/o cultivos celulares.

Identificación mediante Reacción en cadena de la Polimerasa (PCR). Se utilizó la metodología descrita por Park y cols. Se utilizaron los partidores Pgf (5'TGTAGATGACTGATGGTGAAAACC - 3') y C11R (5'ACGTCATCCCCACCTTCCTC - 3'). Los tubos de PCR contenían los siguientes reactivos en $25 \mu \mathrm{l}$ de volumen final: Buffer 10X 2,5 $\mu \mathrm{l}, \mathrm{MgCl} 250 \mathrm{mM} 2 \mu \mathrm{l}$, mezcla equimolar de desoxirribonucleótidos dATP, dGTP, dTTP, dCTP $1 \mu \mathrm{l}, 1 \mu \mathrm{l}$ del partidor Pgf $1 \mu \mathrm{l}$ del partidor $\mathrm{C} 11 \mathrm{R}, 0,2 \mu \mathrm{l}$ de Taq polimerasa 2,5 $\mathrm{U}$, agua Mili $\mathrm{Q}$ $12,3 \mu$ l y $5 \mu$ l de ADN genómico. La amplificación del ADN fue realizada en un termociclador de la siguiente forma: 30 ciclos de desnaturalización a $95^{\circ} \mathrm{C}$ por 30 seg., alineamiento a $60^{\circ} \mathrm{C}$ por $1 \mathrm{~min}$. y extensión $72^{\circ} \mathrm{C}$ por $1,30 \mathrm{~min}$. Además, se contempló un paso inicial de desnaturalización a $95^{\circ} \mathrm{C}$ por 3 min. y una extensión final de $72^{\circ} \mathrm{C}$ por $10 \mathrm{~min}$.

\section{RESULTADOS}

Las características demográficas y clínicas se resumen en la tabla I (número de sujetos de estudio con periodontitis crónica localizada (PCL) y generalizada (PCG), edad (promedio en años + desviación estándar), sexo (F: femenino; $M$ : masculino), profundidad de sondaje y nivel de inserción clínico (NIC) (promedio en $\mathrm{mm}$ y desviación estándar), índice de placa bacteriana y de sangrado (\% promedio y desviación estándar).
De los 37 pacientes muestreados se obtuvo un promedio de $1239,32 \times 10^{-4}$ UFC de los cuales 28 $(75,7 \%)$ fueron positivo para pigmentados de negro (Fig. 7), de ellas el $67,9 \%$ fueron positivas para $P$. gingivalis según técnica de fluorescencia (promedio de 337,92 UFC $\times 10^{-4}$ que corresponde al $24,1 \%$ de colonias por paciente) (Fig. 8). A través de la reacción en cadena de la polimerasa se pudo confirmar que el $84,2 \%$ de las colonias positivas por fluorescencia correspondían a P. gingivalis (Fig. 9).

Al categorizarlos por hábito tabáquico, se obtuvo que el $93,8 \%$ de los pacientes que fuman poseen $P$. gingivalis en su microbiota oral.

\section{Detección de Colonias Pigmentadas de Negro (Lupa Estereoscópica)}

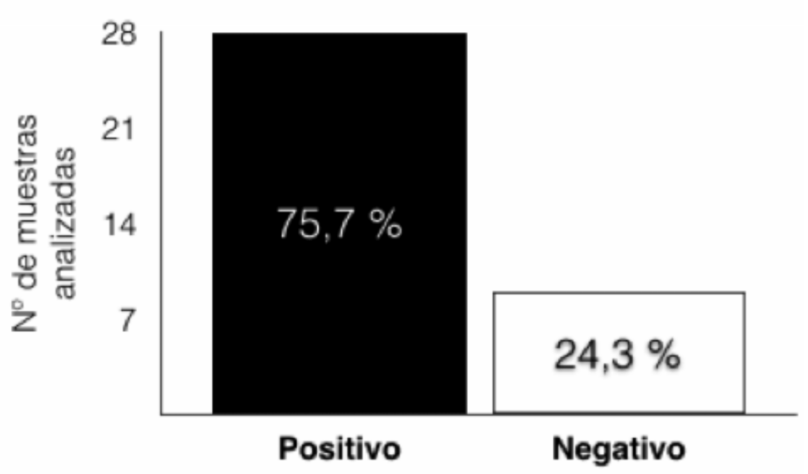

Fig. 7. Detección de colonias pigmentadas de negro. De las 36 muestras analizadas, 28 fueron positivas para colonias pigmentadas de negro con técnica de lupa estereoscópica $(75,7 \%)$.

Tabla I. Características demográficas y clínicas. Número de sujetos de estudio con periodontitis crónica localizada (PCL) y generalizada (PCG), edad (promedio en años + desviación estándar), género ( $F$ : femenino; M: Masculino), profundidad de sondaje y nivel de inserción clínico (NIC) (promedio en mm. y desviación estándar), índice de placa bacteriana y de sangrado (\% promedio y desviación estándar).

\begin{tabular}{lccc}
\hline \multicolumn{1}{c}{ Parámetro } & PCL & PCG & Total \\
\hline Sujetos (n) & 23 & 14 & 37 \\
Edad & $44,8 \pm 13,2$ & $44,8 \pm 11,1$ & $44,8 \pm 12,3$ \\
Sexo (\%) & F: $65,2 \mathrm{M}: 34,8$ & $\mathrm{~F}: 50 \mathrm{M}: 50$ & $\mathrm{~F}: 59 \mathrm{M}: 41$ \\
Prof. de Sondaje (mm) & $5,5+0,7$ & $5,9+0,8$ & $5,6+0,7$ \\
NIC (mm) & $4,9 \pm 1,1$ & $6,1 \pm 1,9$ & $5,4 \pm 1,6$ \\
Índice de Placa (\%) & $97,8 \pm 7,2$ & $94,6 \pm 20,0$ & $96,6 \pm 13,3$ \\
Sangrado al Sondaje (\%) & $95,7+12,3$ & $98,2+6,7$ & $96,6 \pm 10,4$ \\
\hline
\end{tabular}




\section{Detección de $P$. gingivalis a través de Fluorescencia}

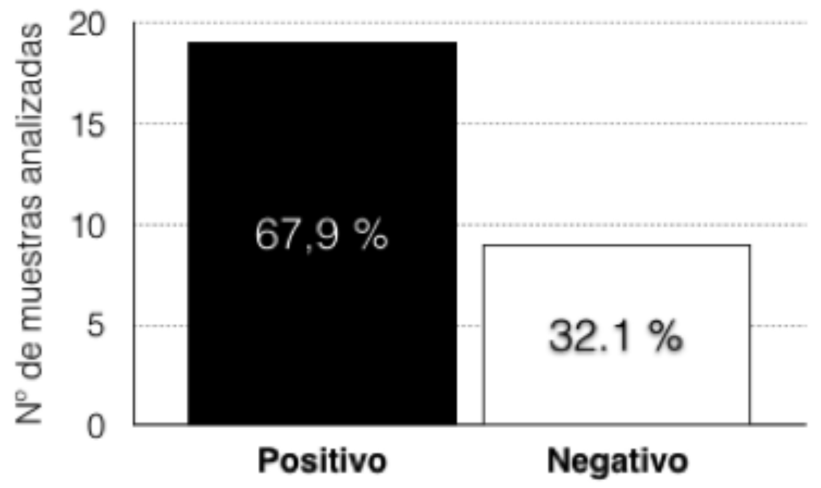

Fig. 8. Detección de $P$. gingivalis a través de técnica de Fluorescencia. De las 28 muestras positivas para pigmentados de negro, 19 fueron positivas para $P$. gingivalis $(67,9 \%)$.

\section{Detección de $P$. gingivalis a través de PCR}

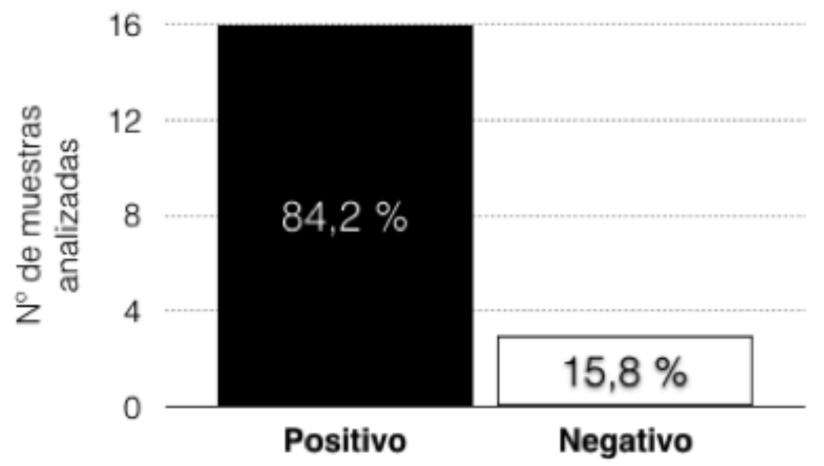

Fig. 9. Detección de colonias pigmentadas de negro. De las 19 muestras positivas para $P$. gingivalis por fluorescencia, 16 fueron también positivas a través de PCR (84.2\%).

\section{DISCUSIÓN}

El presente trabajo detectó una alta prevalencia de $P$. gingivalis $(75,7 \%)$ en los cultivos microbiológicos, encontrando en promedio $24,1 \%$ de UFC por paciente. Esto concuerda con resultados obtenidos en diferentes estudios realizados en Chile y en el mundo. Gajardo et al. (2005) estudiaron 17 pacientes con periodontitis crónica en la Facultad de Odontología de la Universidad de Chile en Santiago, obteniendo un 76,47 \% de $P$. gingivalis en cultivo microbiológico de muestras de placa subgingival. Herrera et al. (2008) estudiaron 37 pacientes con periodontitis crónica, que acudieron a la Facultad de Odontología de la Universidad de Chile en
Santiago, y obtuvo un 83,8 \% de prevalencia en la detección microbiológica de $P$. gingivalis con un 34,01\% de UFC por paciente. Comparó además sus resultados con los obtenidos en Colombia y España donde se obtuvo $65,9 \%$ y $77,8 \%$ respectivamente con una proporción de $5,49 \%$ y $22,1 \%$ de UFC por paciente (Herrera et al.).

López et al. (2004) estudiaron 26 pacientes con periodontitis crónica en la ciudad de Santiago, determinó que de entre las 40 especies bacterianas analizadas, todas, excepto Prevotella intermedia, colonizaron más del $75 \%$ de los sitios y 25 especies colonizaron más del $90 \%$ de los sitios, entre las que se encuentran las bacterias del complejo rojo ( $T$. forsythia y $T$. dentícola), y $P$. gingivalis como la más prevalente.

Goodson et al. (1991) analizaron por sonda de DNA 113 adultos con periodontitis encontrándose un 70 $\%$ de sitios infectados con $P$. gingivalis. Dahlén et al. (1996) analizaron a 20 adultos de una zona rural en Kenya obteniendo un $70 \%$ de $P$. gingivalis, en comparación con otras bacterias del complejo rojo. Herrera et al. (2008) analizaron 84 pacientes con periodontitis crónica, residentes de Bogotá, Colombia, obteniendo un $60,7 \%$ de $P$. gingivalis, mediante cultivo microbiológico en comparación con otras bacterias pertenecientes a placa subgingival.

Takeuchi et al. (2003) analizaron a 35 pacientes con periodontitis crónica en Japón, detectando que $P$. gingivalis fue detectada más frecuentemente en sitios con mayor pérdida en los niveles de inserción clínica (NIC). Lo que concuerda con nuestro estudio, donde $P$. gingivalis es encontrado en pacientes con un promedio en la pérdida del NIC de 5,6 mm (Takeuchi et al.).

Rams et al. (1997) examinaron 1800 muestras subgingivales de pacientes con periodontitis, la proporción media de $P$. gingivalis en la microbiota fue del 23 $\%$, en comparación con otras bacterias periodontales.

Al relacionar el hábito tabáquico con la presencia de $P$. gingivalis, se obtuvo que de los pacientes fumadores un 93,8 \% presenta dicha bacteria en comparación con un $71,4 \%$ de los pacientes no fumadores. De acuerdo al estudio de Ardila Medina et al. (2010) 64,4 \% de 76 pacientes fumadores con periodontitis crónica presentaban $P$. gingivalis. Estos resultados son coincidentes con los obtenidos por nuestro grupo de investigación en el que los pacientes fumadores presentaron menor porcentaje de sangrado al sondaje, mayores profundidades de sondaje y perdida en los niveles de inserción clínica comparados con los no fumadores. Esto 
también se demostró en otros estudios como los de Eggert et al. (2001), Haffajee \& Socransky (2001), van Winkelhoff et al. (2001), Stoltenberg et al. (1993) y Zambon (1996), entre otros.

Es posible concluir que en la población de estudio existe una alta prevalencia de $P$. gingivalis en la microbiota subgingival de los pacientes con periodontitis crónica, lo que ha sido detectado a través de técnicas de microbiología convencional y mediante la reacción en cadena de la polimerasa. Por otra parte, los pacientes fumadores presentaron mayor presencia de $P$. gingivalis que los no fumadores.

ARCE, M; ULLOA, M; POZO, P \& BRAVO-BOWN, J. Porphyromonas gingivalis detection in adult patient with chronic periodontitis. Int. J. Odontostomat., 11(1):13-18, 2017.

SUMMARY: The detection of Porphyromonas gingivalis ( $P$. gingivalis) was performed in adult patients with chronic periodontitis treated at the Clinic of Periodontology in the University of Antofagasta, through of microbiology techniques conventional and molecular biology. Were obtained samples of gingival crevicular fluid with paper cones $\left(\mathrm{N}^{\circ} 30\right)$ for 20 seconds of the more severe periodontal site in each quadrant, 37 patients were studied with chronic periodontitis, subsequent microbiological culture was performed on blood agar and the presence of $P$. gingivalis was detected through conventional microbiology techniques (fluorescence and stereomicroscope) and molecular biology technique (polymerase chain reaction). There was detected with stereomicroscope pigmented black in $75.7 \%$ of the samples, of which $67.9 \%$ were positive for $P$. gingivalis with the fluorescence technique. Through the polymerase chain reaction it was confirmed that $84.2 \%$ of fluorescence positive colonies corresponded to $P$. gingivalis. In the population analyzed can conclude that there is a high prevalence of $P$. gingivalis in the subgingival microbiota of patients with chronic periodontitis which has been detected through techniques of conventional microbiology and by polymerase chain reaction. The smokers had a higher presence of $P$. gingivalis than nonsmokers.

KEY WORDS: Periodontitis chronic, Porphyromonas gingivalis, Polymerase chain reaction

\section{REFERENCIAS BIBLIOGRÁFICAS}

Ardila Medina, C. M.; Alzate Vega, J. \& Guzmán Zuluaga, I. C. Asociación de Aggregatibacter actinomycetemcomitans y microorganismos del complejo rojo con parámetros clínicos de pacientes con periodontitis crónica. Rev. Arch. Méd. Camagüey, 14(3), 2010. Disponible en: http://scielo.sld.cu/ scielo.php?script=sci_arttext\&pid=S1025-02552010000300016

Dahlén, G.; Manji, F.; Baelum, V. \& Fejerskov, O. Black-pigmented
Bacteroides species and Actinobacillus actinomycetemcomitans in subgingival plaque of adult Kenyans. J. Clin. Periodontol., 16(5):305-10, 1989.

Darveau, R. P.; Tanner, A. \& Page, R. C. The microbial challenge in periodontitis. Periodontol. 2000, 14:12-32, 1997.

Eggert, F. M.; McLeod, M. H. \& Flowerdew, G. Effects of smoking and treatment status on periodontal bacteria: evidence that smoking influences control of periodontal bacteria at the mucosal surface of the gingival crevice. J. Periodontol., 72(9):1210-20, 2001.

Gajardo, M.; Silva, N.; Gómez, L.; León, R.; Parra, B.; Contreras, A. \& Gamonal, J. Prevalence of periodontopathic bacteria in aggressive periodontitis patients in a Chilean population. J. Periodontol., 76(2):289-94, 2005.

Goodson, J. M.; Tanner, A.; McArdle, S.; Dix, K. \& Watanabe, S. M. Multicenter evaluation of tetracycline fiber therapy. III. Microbiological response. J. Periodontal Res., 26(5):440-51, 1991.

Haffajee, A. D. \& Socransky, S. S. Relationship of cigarette smoking to the subgingival microbiota. J. Clin. Periodontol., 28(5):377-88, 2001.

Herrera, D.; Contreras, A.; Gamonal, J.; Oteo, A.; Jaramillo, A.; Silva, N.; Sanz, M.; Botero, J. E. \& León, R. Subgingival microbial profiles in chronic periodontitis patients from Chile, Colombia and Spain. J. Clin. Periodontol., 35(2):106-13, 2008.

Holt, S. C. \& Ebersole, J. L. Porphyromonas gingivalis, Treponema denticola, and Tannerella forsythia: the "red complex", a prototype polybacterial pathogenic consortium in periodontitis. Periodontol., 2000, 38:72-122, 2005.

Holt, S. C.; Kesavalu, L.; Walker, S. \& Genco, C. A. Virulence factors of Porphyromonas gingivalis. Periodontol. 2000, 20(1):168-238, 1999.

López, N. J.; Socransky, S. S.; Da Silva, I.; Japlit, M. R. \& Haffajee, A. D. Subgingival microbiota of chilean patients with chronic periodontitis. J. Periodontol., 5(5):717-25, 2004.

Rams, T. E.; Flynn, M. J. \& Slots, J. Subgingival microbial associations in severe human periodontitis. Clin. Infect. Dis., 25 Suppl. 2:S2246, 1997.

Stoltenberg, J. L.; Osborn, J. B.; Pihlstrom, B. L.; Herzberg, M. C.; Aeppli, D. M.; Wolff, L. F. \& Fischer, G. E. Association between cigarette smoking, bacterial pathogens, and periodontal status. J. Periodontol., 64(12):1225-30, 1993.

Takeuchi, Y.; Umeda, M.; Ishizuka, M.; Huang, Y. \& Ishikawa, I. Prevalence of periodontopathic bacteria in aggressive periodontitis patients in a Japanese population. J. Periodontol., 74(10):1460-9, 2003.

van Winkelhoff, A. J.; Bosch-Tijhof, C. J.; Winkel, E. G. \& van der Reijden, W. A. Smoking affects the subgingival microflora in periodontitis. J. Periodontol., 72(5):666-71, 2001.

Zambon, J. J. Periodontal diseases: microbial factors. Ann. Periodontol., 1(1):879-925, 1996.

Zhao, L.; Wu, Y. F.; Meng, S.; Yang, H.; OuYang, Y. L. \& Zhou, X. D. Prevalence of fimA genotypes of Porphyromonas gingivalis and periodontal health status in Chinese adults. J. Periodontal Res., 42(6):511-7, 2007.

Dirección para correspondencia

Dr. Joel Bravo Bown

Av. Argentina 02540 depto. 902

Antofagasta - CHILE

Email: joel.bravo@uantof.cl jbravo@odontologia.uchile.cl

Recibido : 09-07-2016

Aceptado: 23-12-2016 